\title{
SWITCHING ADAPTIVE CONTROL OF AFFINE NONLINEAR SYSTEM
}

\author{
D.V. Efimov \\ Control of Complex Systems Laboratory, \\ Institute of Problem of Mechanical Engineering, \\ Bolshoi av., 61, V.O. , St-Petersburg, 199178 Russia \\ efde@mail.rcom.ru
}

Keywords: adaptive control, disturbances, switching, nonlinear systems.

\begin{abstract}
An switching adaptive control scheme is presented, which provides global asymptotic stability property with respect to state space vector of the plant. In the presence of disturbance these schemes ensure boundedness of all trajectories of the system.
\end{abstract}

\section{Introduction}

This work is devoted to problem of adaptive state feedback stabilisation of affine linearly parameterised system with external disturbances in control channel. Such problem is very popular during last years $[5,6,9,14]$, but, unfortunately, the comprehensive solution is not obtained yet. To take a brief survey into this problem let us consider a task of adaptive stabilisation of the following linear first order system:

$$
\dot{x}=-x+\theta x+u+d,
$$

where $x \in R$ is state variable; $u \in R$ is control; $d \in R$ is external disturbance and $\theta \in \Omega_{\theta} \in R$ is unknown parameter, which belongs to some known compact set, such, that for all $\theta \in \Omega_{\theta}$ inequality $|\theta| \leq \theta_{\max }$ holds for some known positive constant $\theta_{\max }$. It is well known classical solution of task of adaptive stabilisation of (1) for case of disturbance absence $[3,5,7,14]$ :

$$
u=-\widehat{\theta} x, \dot{\hat{\theta}}=\gamma x^{2},
$$

where $\hat{\theta} \in R$ is an estimate of $\theta$. Such solution provides stability of overall system and asymptotic stability with respect to variable $x$. But for case of disturbance $d$ presence such scheme loses overall stability property, and, in general finite time escape phenomena can appear for such adaptive systems [17]. To avoid divergence of variable $\hat{\theta}$ adaptation algorithm (2) can be replaced by $[5,8]$

$$
\dot{\bar{\theta}}=\gamma x^{2}-k \gamma \bar{\theta}
$$

where coefficient $k>0$ introduce additional parametric feedback, which prevents unbounded increasing of variable $\hat{\theta}$. But for unstable systems such solution leads to undesirable response [16] and, additionally, for case of disturbances absence there is an static error in adaptive system [5]. To solve this problem one can use a function of $x$ instead of coefficient $k$ in (3) like it was done in [13]:

$$
\dot{\bar{\theta}}=\gamma x^{2}-\gamma k(x) \hat{\theta}
$$

but such innovation cause in general case a new equilibrium in the system. Another way is dead-zone modification [15, 18] of (2):

$$
\dot{\bar{\theta}}= \begin{cases}\gamma x^{2}, & |x| \geq \Delta ; \\ 0, & |x|<\Delta,\end{cases}
$$

where $\Delta$ is some positive constant, which value depends on disturbance amplitude. Such modification guarantees boundedness of all signal in the system and static error presence for case of disturbance absence. In work [19] more complex adaptive controller was used instead of (2), where additionally upper bound of disturbance is estimated:

$$
\begin{gathered}
u=-\widehat{\theta} x+\widehat{\alpha} \operatorname{sign}(x) ; \\
\dot{\bar{\theta}}=\gamma x^{2} ; \\
\dot{\alpha}=\gamma \widehat{\alpha}^{3 / 2}|x|,
\end{gathered}
$$

where $\hat{\alpha}$ is estimate of amplitude of disturbance $d$. There are several nonlinear adaptive stabilisation algorithms which possess robust stability property with respect to disturbance $[2,9,10,20]$.

In this work another one approach will be utilised, which consists in on-line switching between two adaptation algorithms (2) and (3). First of them provides asymptotic stability property for the plant for case of disturbance absence, while the second one ensures boundedness of all variables of the adaptive system for any bounded disturbances. Such switching will be performed basing only on state of the plant measurements with supposition, that upper bound $\theta_{\max }$ for set $\Omega_{\theta}$ of admissible values of unknown parameters is known. At second section all definitions will be presented, in third section main results will be proved, conclusion finishes the paper.

\section{Definitions and statements}

Let us consider nonlinear dynamic system

$$
\dot{\mathbf{x}}=\mathbf{f}(\mathbf{x})+\mathbf{G}(\mathbf{x})[\boldsymbol{\omega}(\mathbf{x}, t) \boldsymbol{\theta}+\mathbf{u}+\mathbf{d}(t)]
$$

where $\mathbf{x} \in R^{n}$ is state vector, $\mathbf{u} \in R^{m}$ is control; $\mathbf{f}(\mathbf{x})$ and the columns of $\mathbf{G}(\mathbf{x})$ are continuous and locally Lipschitz vector fields on $R^{n}, \mathbf{f}(0)=0 ; \boldsymbol{\omega}(\mathbf{x}, t)$ is known regressor matrix 
function; $\boldsymbol{\theta} \in \Omega_{\theta} \in R^{r}$ is vector of unknown parameters, which values belong to some known compact set, such, that for all $\boldsymbol{\theta} \in \Omega_{\boldsymbol{\theta}}$ inequality $|\boldsymbol{\theta}| \leq \theta_{\max }$ holds for some known positive constant $\theta_{\max } ; \mathbf{d}: R_{\geq 0} \rightarrow R^{p}$ is external disturbance, that is Lebesgue measurable and essentially bounded function of time, it means that

$$
\|\mathbf{d}\|=\operatorname{ess} \sup \{|\mathbf{d}(t)|, t \geq 0\}<+\infty .
$$

Let us introduce main restrictions on properties of system (4).

A s s u mption 1. There exists a function $\rho \in \mathcal{K}$, such, that

$$
|\boldsymbol{\omega}(\mathbf{x}, t)| \leq \rho(|\mathbf{x}|)
$$

for all $\mathbf{x} \in R^{n}$ and all $t \geq 0$.

This supposition rather common and does not mean boundedness of regressor function $\omega$ with respect to variable $\mathbf{x}$. It is said, that function $\rho: R_{\geq 0} \rightarrow R_{\geq 0}$ belongs to class $\mathcal{K}$, if it is strictly increasing and $\rho(0)=0 ; \rho \in K_{\infty}$ if $\rho \in \mathcal{K}$ and $\rho(s) \rightarrow \infty$ for $s \rightarrow \infty$ (radially unbounded). Function $\beta: R_{\geq 0} \times R_{\geq 0} \rightarrow R_{\geq 0}$ is from class $\mathcal{K} \mathcal{L}$, if it belongs to class $\mathcal{K}$ on the first argument (for any fixed second) and strictly decreases to zero on the second (for any fixed first argument).

Assumption 2. There exists a differentiable Lyapunov function $V(\mathbf{x})$, such, that

$$
\alpha_{1}(|\mathbf{x}|) \leq V(\mathbf{x}) \leq \alpha_{2}(|\mathbf{x}|)
$$

for some functions $\alpha_{1}, \alpha_{2} \in K_{\infty}$, and the following inequality holds for all $\mathbf{x} \in R^{n}$

$$
L_{\mathbf{f}} V(\mathbf{x}) \leq-\alpha(|\mathbf{x}|), \alpha \in K_{\infty} .
$$

Expression $L_{\mathbf{f}} V(\mathbf{x})$ denotes scalar Lie derivative of function $V$ with respect to vector field $\mathbf{f}$, term $L_{\mathbf{G}} V(\mathbf{x})$ further will be stated for covector:

$$
L_{\mathbf{f}} V(\mathbf{x})=\partial V(\mathbf{x}) / \partial \mathbf{f} \mathbf{f}(\mathbf{x}), L_{\mathbf{G}} V(\mathbf{x})=\partial V(\mathbf{x}) / \partial \mathbf{x} \mathbf{G}(\mathbf{x}) .
$$

According to Assumption 2 system (4) is globally asymptotically stable for control $\mathbf{u}=0$ with known Lyapunov function $V$ for case of disturbances and parametric uncertainty absence. It is clear, that such property is not sufficient in general to guarantee boundedness of state for any bounded disturbances even in situation, then $\boldsymbol{\theta}=0$.

In next section forward completeness property and input-tostate stability (ISS) property will be used to investigate behaviour of system (4), definitions of these properties and their necessary and sufficient conditions can be found in $[1,21,22$, 23].

\section{Main results}

For system (4) it is possible to use the following control law:

$$
\mathbf{u}=-0.5 L_{\mathbf{G}} V(\mathbf{x})^{T}-\boldsymbol{\omega}(\mathbf{x}, t) \hat{\boldsymbol{\theta}},
$$

where $\widehat{\boldsymbol{\theta}} \in R^{r}$ is an estimate of unknown vector $\boldsymbol{\theta}$. If $\widehat{\boldsymbol{\theta}}=\boldsymbol{\theta}$ then control (5) provides for system (4) ISS property. Indeed, substituting (5) in equation (4) with assumption, that $\widehat{\boldsymbol{\theta}}=\boldsymbol{\theta}$, let us consider time derivative of function $V$ for obtained system:

$$
\begin{aligned}
& \dot{V} \leq-\alpha(|\mathbf{x}|)+L_{\mathbf{G}} V(\mathbf{x})\left[-0.5 L_{\mathbf{G}} V(\mathbf{x})^{T}+\mathbf{d}\right] \leq \\
& \leq-\alpha(|\mathbf{x}|)-0.5\left|L_{\mathbf{G}} V(\mathbf{x})\right|^{2}+\left|L_{\mathbf{G}} V(\mathbf{x})\right||\mathbf{d}| .
\end{aligned}
$$

Using the following simple inequality

$$
\left|L_{\mathbf{G}} V(\mathbf{x})\right||\mathbf{d}| \leq 0.5\left|L_{\mathbf{G}} V(\mathbf{x})\right|^{2}+0.5|\mathbf{d}|^{2},
$$

it is possible to receive:

$$
\dot{V} \leq-\alpha(|\mathbf{x}|)+0.5|\mathbf{d}|^{2},
$$

that according to [23] is equivalent to ISS property. Hence, for case $\widehat{\boldsymbol{\theta}}=\boldsymbol{\theta}$, system (4), (5) possesses asymptotic stability property for vanishing disturbance $\mathbf{d}$ and boundedness of state vector $\mathbf{x}$ for any essentially bounded $\mathbf{d}$.

It is necessary to design an adaptation algorithm for adjusting vector $\hat{\boldsymbol{\theta}}$, such, that for any initial conditions $\mathbf{x}(0) \in R^{n}$, $\widehat{\boldsymbol{\theta}}(0) \in R^{r}$ :

a) solution $(\mathbf{x}(t), \hat{\boldsymbol{\theta}}(t))$ is bounded for any essentially bounded disturbance $\mathbf{d}$;

b) if $\mathbf{d}(t) \equiv 0$ for all $t \geq 0$, then $\mathbf{x}(t)$ should asymptotically converges to zero.

The solution of this problem will be based on two adaptation algorithms construction, which will solve different tasks for situations of disturbance d presence or absence (the conversation is about analogues of algorithms (2) and (3) for system (4)). And after that, a switching algorithm among them will be developed, which under some mild conditions solves posed problem.

First algorithm is a kind of adaptation algorithm (2) for system (4) $[5,6,14]$ :

$$
\dot{\overline{\boldsymbol{\theta}}}=\gamma \boldsymbol{\omega}(\mathbf{x}, t)^{T} L_{\mathbf{G}} V(\mathbf{x})^{T},
$$

where $\gamma$ is a positive design parameter. Main properties of adaptive system with such adaptation algorithm are summarized in the following Lemma [6].

L e m m a 1 . Suppose that for system (4) Assumption 1 and 2 are satisfied. Then system (4), (5), (6) admits properties:

1. Forward completeness for any essentially bounded signal $\mathbf{d}(t)$;

2. Lyapunov stability of $(\mathbf{x}(t), \widehat{\boldsymbol{\theta}}(t))$ and asymptotic attractiveness of $\mathbf{x}(t)$ for $\mathbf{d}(t) \equiv 0, t \geq 0$.

Proof. Let us consider the following Lyapunov function candidate:

$$
U(\mathbf{x}, \widehat{\boldsymbol{\theta}})=V(\mathbf{x})+0.5 \gamma^{-1}(\boldsymbol{\theta}-\widehat{\boldsymbol{\theta}})^{T}(\boldsymbol{\theta}-\widehat{\boldsymbol{\theta}}) .
$$

Its time derivative for the system takes form:

$$
\dot{U} \leq-\alpha(|\mathbf{x}|)+0.5|\mathbf{d}|^{2} .
$$

The last inequality ca be rewritten as

$$
\dot{U} \leq U+0.5|\mathbf{d}|^{2},
$$


that due to properties of function $U$ and work [1] means forward completeness property. For case of disturbance d absence claim of Lemma immediately follows from classical Lyapunov function theory and LaSalle invariance principle.

Thus, algorithm (6) provides for the system asymptotic stability property with respect to variable $\mathbf{x}$ for case of disturbance $\mathbf{d}$ absence, but for any bounded signal $\mathbf{d}$ only forward completeness was proved, opposite to stated in control goals boundedness of solution $(\mathbf{x}(t), \widehat{\boldsymbol{\theta}}(t))$. Such boundedness can be ensured using an analogue of algorithm (3) for system (4) $[5,6,14]$ :

$$
\dot{\tilde{\boldsymbol{\theta}}}=\gamma \boldsymbol{\omega}(\mathbf{x}, t)^{T} L_{\mathbf{G}} V(\mathbf{x})^{T}-\gamma k \widehat{\boldsymbol{\theta}}, k>0 .
$$

Here coefficient $k$ introduce in (6) additional negative feedback, which prevents infinite increasing of variable $\widehat{\boldsymbol{\theta}}$ for any bounded disturbance. Basic properties of such adaptive system are presented in the following Lemma [6].

L e m m a 2. Suppose that for system (4) Assumption 1 and 2 are satisfied. Then system (4), (5), (8) is ISS with respect to extended input $(\mathbf{d}, \boldsymbol{\theta})$.

Pro o f. Time derivative of function (7) takes form for system (4), (5), (8):

$$
\begin{aligned}
\dot{U} \leq & -\alpha(|\mathbf{x}|)-0.5 k(\boldsymbol{\theta}-\widehat{\boldsymbol{\theta}})^{T}(\boldsymbol{\theta}-\widehat{\boldsymbol{\theta}})+ \\
& +0.5 k|\boldsymbol{\theta}|^{2}+0.5|\mathbf{d}|^{2} .
\end{aligned}
$$

That according to properties of function $U$ and [23] implies ISS property for state $(\mathbf{x}, \boldsymbol{\theta}-\widehat{\boldsymbol{\theta}})$ and input $(\mathbf{d}, \boldsymbol{\theta})$.

Thus, adaptation algorithm (8) provides for the system solution $(\mathbf{x}(t), \hat{\boldsymbol{\theta}}(t))$ necessary boundedness property. But from definition of ISS property follows, that for vanishing input $\mathbf{d}$ in common case a static error can appear, which is proportional to $k|\boldsymbol{\theta}|$.

Therefore, making switching to algorithm (6) in the case of disturbance absence and to algorithm (8) if disturbance $\mathbf{d}$ is detected, it is possible to solve proposed adaptive control problem. But, unfortunately, only state of the plant $\mathbf{x}$ is online measured for control purposes. Hence, a supervisor system (i.e. system which will orchestrate of switching among algorithms (6) and (8)) should detect a presence of external disturbances basing only on vector $\mathbf{x}$ measurements.

A way to solve this rather non trivial problem is based on inequality (9) utilising. The main result, that will help us to detect disturbance presence from this inequality, is formulating below.

Prop os ition 1. Suppose that for system (4) Assumption 1 and 2 are satisfied. Then in system (4), (5), (8) for any initial condition $\mathbf{x}(0) \in R^{n}, \widehat{\boldsymbol{\theta}}(0) \in R^{r},|\mathbf{x}(0), \boldsymbol{\theta}-\widehat{\boldsymbol{\theta}}(0)| \leq \kappa$ and for any $\varepsilon>0$ there exists a $T=T(\varepsilon, \kappa)>0$, such, that the following inequality is satisfied:

$$
|\mathbf{x}(t), \boldsymbol{\theta}-\widehat{\boldsymbol{\theta}}(t)| \leq \lambda\left(\theta_{\max }+\|\mathbf{d}\|\right)+\varepsilon,
$$

for all $t \geq T$ and function $\lambda$ from class $\mathcal{K}_{\infty}$.

P r o o f. Inequality (9) can be rewritten as follows:

$$
\begin{aligned}
\dot{U} & \leq-\sigma_{1}(V(\mathbf{x}))+0.5 k|\boldsymbol{\theta}|^{2}+0.5|\mathbf{d}|^{2}- \\
& -\sigma_{2}\left(0.5 \gamma^{-1}(\boldsymbol{\theta}-\widehat{\boldsymbol{\theta}})^{T}(\boldsymbol{\theta}-\widehat{\boldsymbol{\theta}})\right),
\end{aligned}
$$

where functions $\sigma_{1}(s)=\alpha \circ \alpha_{2}^{-1}(s)$ and $\sigma_{2}(s)=\gamma k^{-1} s$ are taken from class $K_{\infty}$. Introducing new function $\sigma(s)=\min _{s}\left\{\sigma_{1}(0.5 s), \sigma_{2}(0.5 s)\right\}$, one can receive:

$$
\dot{U} \leq-\sigma(U)+0.5 k \theta_{\max }^{2}+0.5\|\mathrm{~d}\|^{2},
$$

hence, for all $U \geq \sigma^{-1}\left(0.5 k \theta_{\max }^{2}+0.5\|\mathbf{d}\|^{2}\right)$ inequality $\dot{U} \leq 0$ is true. Thus, for any $\varepsilon \geq 0$, there exists a $T>0$, such, that $U(t) \leq \sigma^{-1}\left(0.5 k \theta_{\max }^{2}+0.5\|\mathbf{d}\|^{2}\right)+\varepsilon$ for all $t \geq T$. But the last fact means, that statement of the Proposition is true for

$$
\begin{aligned}
& \lambda(s)=\delta^{-1}\left(2 \sigma^{-1}\left((k+1) s^{2}\right)\right), \\
& \delta(s)=\min _{s}\left\{\alpha_{1}(2 s), 2 \gamma^{-1} s^{2}\right\} .
\end{aligned}
$$

Note, that result of Proposition 1 is a corollary of uniform asymptotic gain property, which is equivalent to ISS property [23]. By the way, if disturbance $\mathbf{d}$ is absent, then according to Proposition 1 in system (4), (5), (8) plant state trajectory $\mathbf{x}(t)$ and $\hat{\boldsymbol{\theta}}(t)$ enters a neighborhood of the origin $\Xi=\left\{(\mathbf{x}, \widehat{\boldsymbol{\theta}}): \mathbf{x} \in \Theta,|\widehat{\boldsymbol{\theta}}| \leq \theta_{\max }+\lambda\left(\theta_{\max }\right)\right\}$ in finite time. The bounds of this neighborhood depend on value of constant $\theta_{\max }$, which determines admissible values for unknown parameters $\boldsymbol{\theta}$. Of course, the converse in general fails: if in system (4), (5), (8) a trajectory reaches set $\Xi$, then it does not mean, that disturbance $\mathbf{d}$ is zero. This is the main obstacle, that should be handled during further design.

Remark 1. Let us show a way to calculate function $T(\varepsilon, \kappa)$, which was used in Proposition 1. This consideration is based on comparison principle [21]. Note, that the last inequality from proof of Proposition 1 can be rewritten as follows

$$
|(\mathbf{x}, \boldsymbol{\theta}-\widehat{\boldsymbol{\theta}})| \geq \lambda\left(\theta_{\max }+\|\mathbf{d}\|\right) \Rightarrow \dot{U} \leq-0.5 \sigma(U)
$$

Define for any $s \geq 0$ strictly decreasing differential function

$$
\eta(s)=-\int_{1}^{s} \frac{2 d r}{\sigma(r)},
$$

with assumption $\lim _{s \rightarrow 0^{+}} \eta(s)=+\infty$ (in [21] was discussed, that such assumption can be imposed without loosing generality). Then for $(s, r) \in R_{\geq 0} \times R_{\geq 0}$, define

$$
\beta(s, r)=\left\{\begin{array}{l}
0, \quad \text { if } s=0 ; \\
\eta^{-1}(\eta(s)+t), \text { if } s>0 .
\end{array}\right.
$$

In [21] was proved, that $\beta \in K \mathcal{L}$ and for any initial condition $\mathbf{x}\left(t_{0}\right) \in R^{n}, \hat{\boldsymbol{\theta}}\left(t_{0}\right) \in R^{r}$ solution of system (4), (5), (8) admits the following inequality:

$$
U(t) \leq \beta\left(U\left(t_{0}\right), t-t_{0}\right)+\sigma^{-1}\left(k \theta_{\max }^{2}+\|\mathbf{d}\|^{2}\right),
$$

introducing function $\beta_{1}(s, r)=\delta^{-1}(2 \beta(s, r))$ we obtain 


$$
\begin{aligned}
|(\mathbf{x}(t), \boldsymbol{\theta}-\hat{\boldsymbol{\theta}}(t))| \leq & \beta_{1}\left(\left|\left(\mathbf{x}\left(t_{0}\right), \boldsymbol{\theta}-\hat{\boldsymbol{\theta}}\left(t_{0}\right)\right)\right|, t-t_{0}\right)+ \\
& +\lambda\left(\theta_{\max }+\|\mathbf{d}\|\right) .
\end{aligned}
$$

Hence for given $\varepsilon>0$ and $\kappa=\left|\left(\mathbf{x}(0), \widehat{\boldsymbol{\theta}}\left(t_{0}\right)+\theta_{\max }\right)\right|$ it is possible to solve equality

$$
\beta\left(\kappa, T(\varepsilon, \kappa)-t_{0}\right)=\varepsilon,
$$

to obtain desired function $T(\varepsilon, \kappa)$.

Before we proceed with algorithm of supervisor it is worth to note, that in switching systems under acting of disturbances a strange behaviour can appear, which is called chattering regime. Such chattering regime originates from fast switching that can take place in the system due to disturbance presence. Classical definition of differential equations solution does not suit well for dynamic system in chattering regime, hence, some special methods are used in logic-based switching control theory to prevent such regime arising. In this work socalled dwell time technique will be used [11, 12], which is traditionally conformed to linear switching control systems only due to finite time escape phenomena in nonlinear systems. Here to avoid this obstacles in nonlinear systems we will prove forward completeness property of whole system, as it was done in [2].

For system (4), (5) we will use the following switching adaptive algorithm:

$$
\begin{gathered}
\dot{\overrightarrow{\boldsymbol{\theta}}}=\gamma \mathbf{F}_{i}(\mathbf{x}, \widehat{\boldsymbol{\theta}}), i=1,2 ; \\
\mathbf{F}_{1}(\mathbf{x}, \widehat{\boldsymbol{\theta}})=\boldsymbol{\omega}(\mathbf{x}, t)^{T} L_{\mathrm{G}} V(\mathbf{x})^{T}, \\
\mathbf{F}_{2}(\mathbf{x}, \widehat{\boldsymbol{\theta}})=\boldsymbol{\omega}(\mathbf{x}, t)^{T} L_{\mathbf{G}} V(\mathbf{x})^{T}-k \widehat{\boldsymbol{\theta}},
\end{gathered}
$$

where $\gamma>0$ and $k>0$ as above are design parameters, variable $i$ determines current right hand side of adaptation algorithm (11). As it follows from (11), for $i=1$ algorithm (11) transforms to (6), and for $i=2$ this algorithm switches to (8). So, supervision algorithm can be described as follows:

$$
\begin{aligned}
& i(t)=\left\{\begin{array}{l}
i\left(t_{k}\right) \text { if } \tau<\tau_{D} ; \\
\left\{\begin{array}{l}
1, \text { if }(\mathbf{x}(t), \widehat{\boldsymbol{\theta}}(t)) \in \Xi ; \\
2, \text { if }(\mathbf{x}(t), \widehat{\boldsymbol{\theta}}(t)) \notin \Xi ;
\end{array} \text { if } \tau \geq \tau_{D} ;\right.
\end{array}\right. \\
& \dot{\tau}=1, \tau\left(t_{k}\right)=0 \text {, }
\end{aligned}
$$

where auxiliary variable $\tau$ represents internal supervisor timer dynamic, $\tau_{D}>0$ is dwell time constant and $t_{k}$, $k=0,1,2, \ldots$ are moments of switching (moments then signal $i(t)$ changes its value), $k$ is number of current switching. The operating of algorithm (12) can be explaining in the following way: internal timer $\tau$ is initialised to zero after each switching, while $\tau<\tau_{D}$ signal $i(t)$ does not change its value. Dwell time presence in algorithm (12) help us to prevent fast switching arising in the system (4), (5), (11), (12). After dwell time signal $i(t)$ can be set up to 1 , if $|\mathbf{x}(t)| \leq \lambda\left(\theta_{\max }\right)$, $|\widehat{\boldsymbol{\theta}}| \leq \theta_{\max }+\lambda\left(\theta_{\max }\right)$ and disturbance $\mathbf{d}$ absence it is supposed; signal $i(t)$ will be set up to 2 , if disturbance is detecting from condition $|\mathbf{x}(t)|>\lambda\left(\theta_{\max }\right) \quad$ or $|\widehat{\boldsymbol{\theta}}|>\theta_{\max }+\lambda\left(\theta_{\max }\right)$. The whole system properties are stated in the following Theorem.

Th e o r e m 1. Suppose that for system (4) Assumption 1 and 2 are satisfied. Then system (4), (5), (11), (12) admits properties:

1. Global boundedness of solution $\widetilde{\mathbf{x}}(t)=(\mathbf{x}(t), \widehat{\boldsymbol{\theta}}(t))$ for any essentially bounded signal $\mathbf{d}(t)$ :

$$
\begin{gathered}
|(\mathbf{x}(t), \boldsymbol{\theta}-\hat{\boldsymbol{\theta}}(t))| \leq \beta_{1}\left(U_{\max }, 0\right)+\lambda\left(\theta_{\max }+\|\mathbf{d}\|\right), \\
U_{\max }=\left[\begin{array}{c}
\alpha_{2}\left(\max \left(\lambda\left(\theta_{\max }\right),|\mathbf{x}(0)|\right)\right)+ \\
+\gamma^{-1}\left(\theta_{\max }+\lambda\left(\theta_{\max }\right)\right)+0.5\|\mathbf{d}\|^{2}
\end{array}\right] e^{\tau_{D}} ;
\end{gathered}
$$

2. Global asymptotic attractiveness of $\mathbf{x}(t)$ for $\mathbf{d}(t) \equiv 0$, $t \geq 0$.

P r o o f. Let us at first investigate continuity property of system (4), (5), (11), (12) solution $\widetilde{\mathbf{x}}(t)$. Note, that according to Lemmas $1-2$, system is forward complete for $i=1$ and ISS for $i=2$, thus, it is possible to conclude, that for each value of signal $i(t) \in\{1,2\}$ solution of the system $\widetilde{\mathbf{x}}$ is defined as long, as we need. Additionally, at each switching time $t_{k}$, $k=1,2, \ldots$ functions $\mathbf{F}_{i}, i=1,2$ have finite values, presence of dwell time in algorithm (12) bounds number of switching $N_{\left[t_{A}, t_{B}\right]}$ on any time interval $\left[t_{A}, t_{B}\right]$ in the following obvious way:

$$
N_{\left[t_{A}, t_{B}\right]} \leq \frac{t_{B}-t_{A}}{\tau_{D}}+1
$$

Consequently, function $\mathbf{F}_{i(t)}(\widetilde{\mathbf{x}}) \in \mathbf{F}(\widetilde{\mathbf{x}})$, where $\mathbf{F}$ is some differential inclusion with convex compact and non empty values for each $\widetilde{\mathbf{x}} \in R^{n+r}$, which is upper semicontinuous for all $\widetilde{\mathbf{x}} \in R^{n+r}$. Then according to $[4,24]$ for each $\widetilde{\mathbf{x}}(0) \in R^{n+r}$ system (4), (5), (11), (12) has a maximal solution $\widetilde{\mathbf{x}}(t)$, which is absolutely continuous function of time and defined at least locally on interval $\left[0, T_{\tilde{\mathbf{x}}}\right)$. But according to Lemmas $1-2$, this solution is defined for all $t \geq 0$. Now we are ready to base boundedness property, stated at first point of the Theorem.

Suppose, that there exists some $T_{1}>0$, such, that $i(t)=$ const for all $t \geq T_{1}$. Then $\widetilde{\mathbf{x}}(t)$ is bounded, indeed, if $i(t)=1$, then by construction $\widetilde{\mathbf{x}}(t) \in \Xi$ for all $t \geq T_{1}$; in opposite case, if $i(t)=2$, then according to Lemma 2 system admits ISS property and solution $\widetilde{\mathbf{x}}(t)$ possesses inequality (10). Thus, if such time $T_{1}$ exists, then first point of the Theorem is proven. Hence, further we assume, that there is no such time $T_{1}$ and in this situation an infinite sequence of time intervals is appeared $\mathcal{T}=\left\{\left[t_{k}, t_{k+1}\right), k=0,1,2, \ldots\right\}$, where $t_{k}$ are moments of algorithm (12) switching and $t_{0}=0$. Without loosing generality we can suppose, that for all odd values of index $k$ on time intervals $\left[t_{k}, t_{k+1}\right)$ value of $i(t)$ equals to 1 , for all even values of index $k$ it equals to 2 . Let us consider time inter- 
vals, where $i(t)=1$. At beginning of these time intervals the following inequality is satisfied:

$$
\begin{gathered}
\left|\mathbf{x}\left(t_{k}\right)\right| \leq \max \left(\lambda\left(\theta_{\max }\right),|\mathbf{x}(0)|\right), \\
\left|\hat{\boldsymbol{\theta}}\left(t_{k}\right)\right| \leq \theta_{\max }+\lambda\left(\theta_{\max }\right),
\end{gathered}
$$

here it is assumed, that $|\widehat{\boldsymbol{\theta}}(0)| \leq \theta_{\max }$. Starting from this initial conditions set a trajectory $\widetilde{\mathbf{x}}(t)$ can leave set $\Xi$. Note, that by construction of algorithm (12) the maximum time before switching in the second position, while $\widetilde{\mathbf{x}}(t) \notin \Xi$, is limited by constant $\tau_{D}$. As it follows from Lemma 1, system is forward complete at this time interval and inequality

$$
\dot{U} \leq U+0.5\|\mathbf{d}\|^{2}
$$

holds. It is a linear differential inequality, which solution possesses an estimate:

$$
U(t) \leq\left[U\left(t_{k}\right)+0.5\|\mathbf{d}\|^{2}\right] e^{t-t_{k}} .
$$

From (13) $U\left(t_{k}\right) \leq \alpha_{2}\left(\left|\mathbf{x}\left(t_{k}\right)\right|\right)+\gamma^{-1}\left(\theta_{\max }+\lambda\left(\theta_{\max }\right)\right)$, then for all $t \in\left[t_{k}, t_{k+1}\right)$ and for each odd values of index $k$ function $U(t)$ admits inequality:

$$
U(t) \leq\left[\begin{array}{c}
\alpha_{2}\left(\max \left(\lambda\left(\theta_{\max }\right),|\mathbf{x}(0)|\right)\right)+ \\
+\gamma^{-1}\left(\theta_{\max }+\lambda\left(\theta_{\max }\right)\right)+0.5\|\mathbf{d}\|^{2}
\end{array}\right] e^{\tau_{D}} .
$$

Inequality (14) should be understood taking in mind, that length of time interval $\left[t_{k}, t_{k+1}\right)$ can be bigger than $\tau_{D}$, but length of time, during that trajectory $\widetilde{\mathbf{x}}(t)$ leaves set $\Xi$, can not exceed constant $\tau_{D}$. Hence, on time intervals with $i(t)=1$ solution of system (4), (5), (11), (12) is bounded. Further, let us consider time intervals with even values of index $k$, where $i(t)=2$. Rewrite inequality (10) for function $U$ like it was done in Remark 1 ,

$$
\begin{aligned}
U(t) & \leq \beta\left(U\left(t_{k}\right), t-t_{k}\right)+\sigma^{-1}\left(k \theta_{\max }^{2}+\|\mathbf{d}\|^{2}\right) \leq \\
& \leq \beta\left(U\left(t_{k}\right), 0\right)+\sigma^{-1}\left(k \theta_{\max }^{2}+\|\mathbf{d}\|^{2}\right) .
\end{aligned}
$$

It is possible to conclude, that on each time intervals with $i(t)=2$ solution of system (4), (5), (11), (12) is bounded. Substituting (14) in the last inequality we can receive stated in Theorem upper estimate for $\widetilde{\mathbf{x}}(t)$.

Let us substantiate second point of the Theorem. From Proposition 1 follows, that if $\mathbf{d}(t) \equiv 0$ for all $t \geq 0$, then for case $i(t)=2$ there exists a $T \geq 0$, such, that $\widetilde{\mathbf{x}}(t) \in \Xi, t \geq T$ and algorithm (12) should switch at this time to first position, then also according to proof of Proposition 1 inequality

$$
U(t) \leq \sigma^{-1}\left(k \theta_{\max }^{2}\right)
$$

should be satisfied for $t \geq T$. Expected value of time $T$ can be calculated using result of Remark 1 . It is easy to see, that $\widetilde{\mathbf{x}}(t) \in \Xi$ for all $t \geq 0$ for case $i(t)=1$ with absent disturbances. Indeed, from Lemma 1 in this situation the following inequality holds:

$$
\dot{U} \leq-\alpha(|\mathbf{x}|),
$$

hence, value of function $U(t)$ does not increase:

$$
U(t) \leq U\left(t_{k}\right), t \geq t_{k},
$$

where $t_{k}$ is a time to last switching of signal $i(t)$ in first position, but by continuity due to previous discussion $U\left(t_{k}\right) \leq \sigma^{-1}\left(k \theta_{\max }^{2}\right)$. It means that

$$
|\mathbf{x}(t), \boldsymbol{\theta}-\widehat{\boldsymbol{\theta}}(t)| \leq \lambda\left(\theta_{\max }\right)
$$

for all $t \geq t_{k}$ and condition $\widetilde{\mathbf{x}}(t) \in \Xi$ holds for all such time.

It is worth to stress that upper bound estimate for solution of switching adaptive system (4), (5), (11), (12) is bigger than for ISS system (4), (5), (8) presented in expression (10):

$$
|(\mathbf{x}(t), \boldsymbol{\theta}-\widehat{\boldsymbol{\theta}}(t))| \leq \beta_{1}\left(\left|\left(\mathbf{x}\left(t_{0}\right), \boldsymbol{\theta}-\widehat{\boldsymbol{\theta}}\left(t_{0}\right)\right)\right|, 0\right)+\lambda\left(\theta_{\max }+\|\mathbf{d}\|\right) .
$$

But it is a price for global attractiveness property of variable $\mathbf{x}(t)$ for case disturbance $\mathbf{d}$ missing. Augmenting value of design parameter $\gamma$ and decreasing value of another design parameter $\tau_{D}$ it is possible to bring near both estimate for robust adaptive and switching adaptive systems. Another constant, which influences on value of upper bound estimate of switching adaptive system solution is gain $k$, that small values are preferable.

Remark 2. Algorithm (12) has one disadvantage, which deals with continuous decision making for $\tau \geq \tau_{D}$. In other words, for $\tau \geq \tau_{D}$ algorithm (12) should immediately switch signal $i(t)$ in another position at precisely that moment of time, where corresponded condition for switching is satisfied $((\mathbf{x}(t), \widehat{\boldsymbol{\theta}}(t)) \in \Xi$ or $(\mathbf{x}(t), \widehat{\boldsymbol{\theta}}(t)) \notin \Xi)$. But on practice, to make such decision a some time delay is necessary. This fact is connected with realization of algorithm (12), typically it is a kind of computer system, which is functioning with some sample time $\tau_{C}>0$, that is spent on measurements, calculating of switching condition and so on. Thus, algorithm (12) in such situation should be rewritten as follows:

$$
\begin{aligned}
& i(t)=\left\{\begin{array}{l}
i\left(t_{k}\right) \text { if } \tau<\tau_{D}, \tau \neq \tau_{D}+z \tau_{C} ; \\
\left\{\begin{array}{l}
1, \text { if }(\mathbf{x}(t), \widehat{\boldsymbol{\theta}}(t)) \in \Xi ; \\
2, \text { if }(\mathbf{x}(t), \widehat{\boldsymbol{\theta}}(t)) \notin \Xi ;
\end{array} \text { if } \tau=\tau_{D}+z \tau_{C} ;\right.
\end{array}\right. \\
& \dot{\tau}=1, \tau\left(t_{k}\right)=0, z=0,1,2, \ldots
\end{aligned}
$$

In supervision algorithm (15) signal $i(t)$ can change its value only in specified time moments, where internal time $\tau=\tau_{D}+z \tau_{C}, \quad z=0,1,2, \ldots$ All other time before switchings value of signal $i(t)$ can not be changed. It is clear, that for small values of constant $\tau_{C}$ applicability conditions of algorithms (12) and (15) are the same. If one would use algorithm (15) to prove result like was stated in Theorem 1, then minor modifications are needed.

\section{Conclusion}

An switched adaptive algorithm is proposed, which can provide boundedness of system solution with global attractiveness property for plant state. Construction of switched algorithm additionally needs only knowledge about constant $\theta_{\max }$, that defines upper bound of set of admissible values for vector of unknown parameters $\boldsymbol{\theta}$. Detection of disturbance $\mathbf{d}$ pres- 
ence in the system is performed basing on on-line measurements of plant state $\mathbf{x}$. Though on complex formulas for supervision algorithm (12) or (15), practical implementation of obtained solution does not meet significant difficulties, as it was shown during computer simulation phase.

\section{Acknowledgements}

This work is partly supported by grant 03-01-06373 of Russian Foundation for Basic Research.

\section{References}

[1] Angeli D., Sontag E.D. Forward completeness, unboundedness observability, and their Lyapunov characterizations. Systems and Control Letters, 38, pp. 209 - 217, (1999).

[2] Bobtsov A.A., Efmov D.V. Adaptive control of affine nonlinear system with functional uncertainty. Proc. IEEE ECC 2003, (2003).

[3] Byrnes C.I., Willems J.C. Adaptive Stabilization of Multivariable Linear Systems. Proc. IEEE CDC 84, Las Vegas, pp. 1574 - 1577, (1984).

[4] Filippov A.F. Differential equations and discontinuous righthand sides. Kluwer Academic Publishers, (1988).

[5] Fomin V.N., Fradkov A.L., Yakubovich V.A. An adaptive control of dynamic plants. Moscow: Science, 448 p., (1981). (in Russian)

[6] Fradkov A.L., Miroshnik I.V., Nikiforov V.O. Nonlinear and adaptive control of complex systems. Kluwer Academic Publishers, 528 p., (1999).

[7] Ilchmann A. Non-Identifier-Based Adaptive Control of Dynamical Systems: A Survey. IMA J. Math. Contr. Info., 8, pp. 321 - 366, (1991).

[8] Ioannou P., Kokotovic P. Instability Analysis and Improvement for Robustness of Adaptive Control. Automatica, 20, pp. 583 - 594, (1984).

[9] Krstic M., Kanellakopoulos I., Kokotovic P. Nonlinear and Adaptive Control Design. New York: John Wiley, (1995).

[10] Mareels I.M.Y., Penfold H.B., Evans R.J. Controlling Nonlinear Time-varying Systems Via Euler Approximations. Automatica, 28, pp. 681 - 696, (1992).

[11] Morse A.S. Control using logic-based switching. In: Trends in control (A. Isidory (Ed.)), Springer-Verlag, pp. $69-113,(1995)$.

[12] Morse A.S. Supervisory control of families of linear setpoint controllers - Part 1: exact matching. IEEE Trans. Aut. Contr., 42, pp. 171 - 187, (1996).

[13] Narendra K., Annaswamy A. A New Adaptive Law for Robust Adaptive Control Without Persistent Excitation. IEEE Trans. Aut. Contr., AC-32, pp. 134 - 145, (1987).

[14] Narendra K., Annaswamy A. Stable Adaptive Systems. Englewood Cliffs: Prentice Hall, (1989).

[15] Ortega R., Lozano-Leal R. A Note on Direct Adaptive Control of Systems with Bounded Disturbances. Automatica, 23, pp. 253 - 254, (1987).

[16] Polderman J.W., Mareels I.M.Y. High Gain Adaptive
Control Revisited: First and Second Order Case. Proc. IEEE CDC 99, Phoenix, pp. 3329 - 3333, (1999).

[17] Rohrs C.E., Velavani L., Athans M., Stein G. Robustness of Continuous-Time Adaptive Control Algorithms in the Presence of Unmodeled Dynamics. IEEE Trans. Aut. Contr., AC-30, pp. 881 - 889, (1985).

[18] Samson C. Stability Analysis of Adaptively Controlled Systems Subject to Bounded Disturbances. Automatica, 19, pp. $81-86$, (1983).

[19] Sane H.S., Roup A.V., Bernstein D.S., Sussmann H.J. Adaptive stabilisation and disturbance rejection for firstorder systems. Proc. IEEE ACC 2002, (2002).

[20] Skafidas E., Fradkov A., Evans R.J., Mareels I.M.Y. Trajectory-Approximation-Based Adaptive Control for Nonlinear Systems Under Matching Conditions. Automatica, 34, pp. 287 - 299, (1998).

[21] Sontag E.D. Smooth stabilization implies coprime factorization. IEEE Trans. Aut. Contr., 34, pp. 435 - 443, (1989).

[22] Sontag E.D., Wang Y. Various results concerning set input-to-state stability. Proc. IEEE CDC 95, IEEE Publications, pp. 1330 - 1335, (1995).

[23] Sontag E.D., Wang Y. On characterization of the input to state stability property. Systems and Control Letters, 24, pp. $351-359$, (1995).

[24] Teel A., Praly L. Results on converse Lyapunov functions from class- $K \mathcal{L}$ estimates. Proc. IEEE CDC 99, IEEE Publications, pp. 2545 - 2550, (1999). 